\title{
The Chemistry of Nucleation: In situ Pair Distribution Function analysis of secondary building units during UiO-66 MOF formation
}

\author{
Hui $X u,{ }^{[a, b]}{ }^{\circ}$ Sanna Sommer, ${ }^{[a] \ddagger}$ Nils Lau Nyborg Broge, ${ }^{[a]}$ Junkuo Gao, ${ }^{[c]}$ and Bo. B. Iversen ${ }^{\star[a]}$
}

1 Abstract: The concept of Secondary Building Units (SBUs) is central to a $3 / 4$

2 science on metal-organic frameworks (MOFs), and they are widely used to 5

3 design new MOF materials. However, the presence of SBUs during MOF6

4 formation remains controversial, and the formation mechanism of $\mathrm{MO}_{3} 7$

5 remains unclear due to limited information about the evolution 38

6 prenucleation cluster structures. Here in situ Pair Distribution Function

7 (PDF) analysis was used to probe UiO-66 formation under solvothermal

8 conditions. The expected SBU - a hexanuclear zirconium cluster - is present?

9 in the metal salt precursor solution. Addition of organic ligands results in 43

10 disordered structure with correlations up to $23 \AA$ resembling crystallin $\$ 4$

11 UiO-66. Heating leads to fast cluster aggregation, and further growth an $\$ 5$

12 ordering results in the crystalline product. Thus, SBUs are present alread46

13 at room temperature and act as building blocks for MOF formation. Th47

14 proposed formation steps provide insight for further development of MQ\$8

15 synthesis.

16 Introduction

17 Metal Organic Frameworks (MOFs) constitute a fascinating clas 54

18 of crystalline porous materials with applications in many differe 5

19 areas such as gas storage/separation, luminescent sensing, drug 6

20 delivery and photocatalysis. ${ }^{[1]}$ The idea of "designing" LEGOT5 7

21 like MOF structures is generally considered to involve choosing 58

22 metal polyhedron unit, or so-called secondary building unit (SB $\overline{9}, 9$

23 with known coordination preferences, and then link these wif 0

24 organic ligands to form predictable three-dimensional fram 61

25 works. ${ }^{[2]}$ This is currently possible to a limited extent, despite the 2

26 relatively poor evidence for the actual atomic-scale mechanisn 3

27 of MOF formation in solvothermal synthesis. This gap $\quad 64$

28 knowledge hampers both the preparation of new MOFs as well 285

29 the ability to precisely control their crystal growth and morpholog 6.6

30 Research on the mechanism of crystal nucleation and grow 67

31 is an important area in physics, chemistry, materials science arfel 8

32 environmental research. ${ }^{[3]}$ As an example crystal nucleation arfer9

33 growth plays an important role for controlling the morphology an 70

[a] Dr. H. Xu, S. Sommer, N. L. N. Broge, Prof. Dr. B. B. Iversen Center for Materials Crystallography

Department of Chemistry and iNANO, Aarhus University

Langelandsgade 140, 8000 Aarhus (Denmark)

E-mail: bo@chem.au.dk

[b] Dr. H. Xu

College of Materials Science and Engineering, China Jiliang

University, Hangzhou 310018, P. R. China

[c] Prof. Dr. J. Gao

College of Materials and Textiles, Zhejiang Sci-Tech University, Hangzhou 310018, P. R. China

$\ddagger \quad$ Both authors have equal contribution to this paper

Supporting information for this article is given via a link at the end of 84 the document. facets in metal nanocatalysts, ${ }^{[4]}$ and it is also crucial in our daily life e.g. for the formation of atmospheric particles. ${ }^{[5]}$ Clearly, a proper understanding of prenucleation processes as well as the mechanisms controlling nanocrystal properties is highly desired. The prevailing nucleation and growth models range from classical LaMer and Johnson-Mehl-Avarami models to newer prenucleation cluster and oriented attachment models. ${ }^{[6]}$ These models have been used to describe nucleation and growth in many different cases based on a simplified notion of "particles" or "monomers", but with limited consideration given to the atomic scale nature of a system. In general, there is limited understanding of the underlying "chemistry" of nucleation and growth processes.

The study of MOF formation has been based on different techniques with different strengths, which when used in conjunction overcome their individual shortcomings. ${ }^{[7]}$ In situ energy-dispersive powder $\mathrm{X}$-ray diffraction (PXRD) is an appropriate technique to investigate the formation and evolution of crystalline MOF phases. ${ }^{[8]}$ Interesting metastable intermediates and interconversion of MOFs were observed through in situ PXRD measurements, which provided a comprehensive picture of MOF crystallization. ${ }^{[7,9]}$ Small Angle X-ray Scattering (SAXS) was used to probe the precursor clusters and the nanocrystal formation mechanisms in order to overcome the limitations of PXRD for analyzing the processes occurring before crystallization. However SAXS is not able to reveal atomistic structural details of the nucleation and growth processes. ${ }^{[10]}$ Therefore, MOF formation remains a black box without atomic scale insight.

SBUs are the conceptual basic building units and their connectivity greatly influences final MOF topology. ${ }^{[11]}$ A few pioneering studies based on a two step crystal engineering strategy relied upon first preparing the SBUs and then constructing tunable MOF structures, ${ }^{[12]}$ but due to inherent limitations of the techniques used, the cluster structure of MOF precursor species in homogenous solution is still unknown. Furthermore, the actual formation of MOF SBUs in solution prior to, or during, crystallization continues to be a source of contention. ${ }^{[7]}$ The pioneering work by Ferey utilized ex situ EXAFS and NMR to verify the SBU hypothesis, and their findings indicated that SBUs form prior to MOF crystallization, and can be retained during MOF synthesis. ${ }^{[13]}$ On the other hand, the AFM work by Shoaee on HKUST-1 does not support this conclusion, and rather suggests that monomers and isolated ligands are the basic growth units. ${ }^{[14]}$ The techniques employed by both these studies are constrained by an inability to provide direct structural evidence for the presence of SBUs prior to and during MOF crystallization, and therefore cannot definitively answer if SBUs contribute to MOF formation or not.

In situ X-ray total scattering is a powerful tool for studying materials formation mechanisms under solvothermal synthesis conditions, as it allows extraction of structural information from amorphous materials (including gases and liquids), short-range 
1 order nano-particles, and long-range order crystalline particles.

2 Pair distribution function (PDF) analysis, obtained by Fourier

3 transformation of the total scattering structure factor $S(Q)$, provide

4 a histogram of all interatomic distances in the sample

5 ("fingerprint"). ${ }^{[15]}$ In situ total scattering has previously been used

6 to reveal a rich chemistry in the nucleation and growth of inorganic

7 materials such as $\mathrm{CeO}_{2}, \mathrm{SnO}_{2}, \mathrm{ZrO}_{2}, \mathrm{Fe}_{2} \mathrm{O}_{3}, \mathrm{WO}_{3}$ and $\mathrm{ZnWO}_{4} \cdot{ }^{[16]}$

8 In the field of MOFs, work by Chapman et al. utilized PDF

9 analysis to probe local structural transitions of $\mathrm{M}_{6} \mathrm{O}_{8}$ nodes during

10 heating UiO-66 and NU-1000 in dry powder form. ${ }^{[17]}$ Recently, the

11 pioneering work by Billinge et al. combined multiple techniques

12 such as in situ PDF, mass spectrometry and DFT calculations to

13 reveal the early stage structural development of prototypical

14 zeolitic imidazolate framework in solution. ${ }^{[18]}$ These results

15 showed the promise of PDF analysis of SBU structures, and

16 inspired the present in situ study of the solvothermal formation of

17 UiO-66. Since the SBUs in MOFs consist of metal-oxide clusters,

18 we chose a relatively heavy metal MOF system to generate strong

19 X-ray scattering. This facilitates the possibility to catch a glimpse

20 of the fundamental chemical transformations taking place before

21 and during the initial nucleation stage as well as the evolution of

22 the cluster structure during early growth. Here, we present an in

23 situ PDF study on the solvothermal synthesis of UiO-66.

\section{Results and Discussion}

Cluster formation in the UiO-66 solution before addition $5 f 7$ the organic linker

We first study the nature of the solution of the metal source

28 before addition of the organic linker. As shown in Figure 1a, the

29 most prominent features of the dissolved $\mathrm{Zr}(\mathrm{IV})$ precursor $\left(\mathrm{ZrCl}_{\mathrm{O}}\right)_{1}$

30 in $\mathrm{DMF}$ and $\mathrm{HCl}$ solution $(\mathrm{PH}=1.8)$ are the intense PDF peaks

$312.2,3.5$ and $5.0 \AA$. No correlations are observed above $8 \AA 3$

32 (Figure 1(a)), which suggest that no clusters larger than $8 \AA$ exight 4

33 in the metal precursor solution. This led us to consider that the

34 species in this metal salt solution may be the UiO-66 SB 66

$35 \mathrm{Zr}_{6}(\mathrm{O})_{4}(\mathrm{OH})_{4}$ structure, which is a $\mathrm{Zr}_{6}$-Octahedron bridged by $\mu \mathrm{C} 7$

$36 \mathrm{O}$ and $\mu 3-\mathrm{OH}$ groups, and with main $\mathrm{Zr} \cdots \mathrm{O}$ and $\mathrm{Zr} \cdots \mathrm{Zr}$ distances

37 of $2.2,3.5$ and $5.0 \AA$, respectively. ${ }^{[19]}$ The PDF is unable $\overbrace{}^{\circ}$

38 differentiate whether there is hydrogen present because of the

39 weak scattering from hydrogen. Thus, the UiO-66 SBU structure 1

40 was isolated from the crystal structure (ICSD-4512072), $[19]_{7}^{1}$

41 yielding a hexanuclear $\mathrm{Zr}$ cluster $\left(\mathrm{Zr}_{6}(\mathrm{O})_{8}(\mathrm{O})_{24}\right)$, which has eight

42 faces of a regular octahedron bridged by eight $\mu 3$-oxygen atoms 4

43 and the cluster is terminated by 24 oxygen atoms from solvent 5

$44 \mathrm{H}_{2} \mathrm{O}$ or DMF to ensure a saturated coordination, as shown

45 Figure $1 \mathrm{~b}$. The main atom distances in this SBU includes the $\mathrm{Z}_{7} 7$

$46 \mathrm{O}$ bond at approximately $2.2 \AA$, the $\mathrm{Zr} \cdots \mathrm{Zr}$ distances across the 8

47 edges of the octahedron at approximately $3.5 \AA$, and the diagonal

$48 \mathrm{Zr} \cdots \mathrm{Zr}$ distances inside the SBU at approximately $5.0 \AA$. The

$49 \mathrm{Zr} \cdot \mathrm{Zr}$ distances at $3.5 \AA$ and $5.0 \AA$ are associated with 12 and $\mathcal{8} 1$

50 equivalent distances, respectively, which determines the PD 1

51 peak area. The hexanuclear SBU was modelled against the PDE3

52 data using the program DiffyCMI, ${ }^{[20]}$ and acceptable fits were 84

53 obtained both for $\mathrm{ZrCl}_{4}$ in DMF-HCl solution (Figure 1(a)) as wक्षी

54 as for $\mathrm{ZrCl}_{4}$ in DMF- $\mathrm{H}_{2} \mathrm{O}$ solution (see supporting informationf

55
(A)

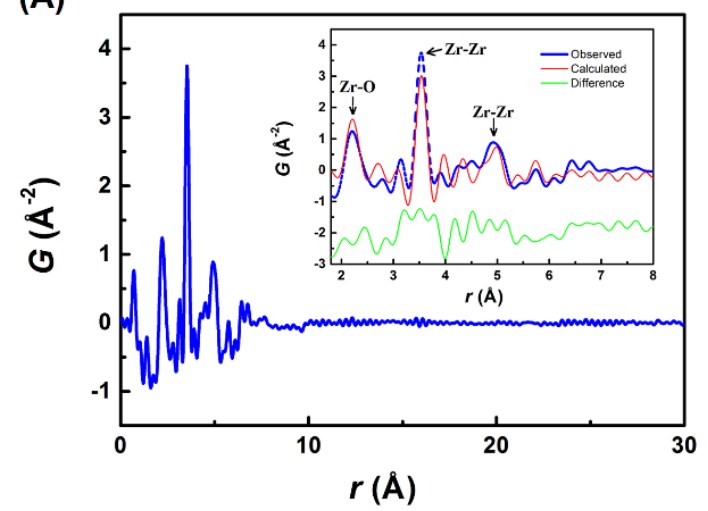

(B)

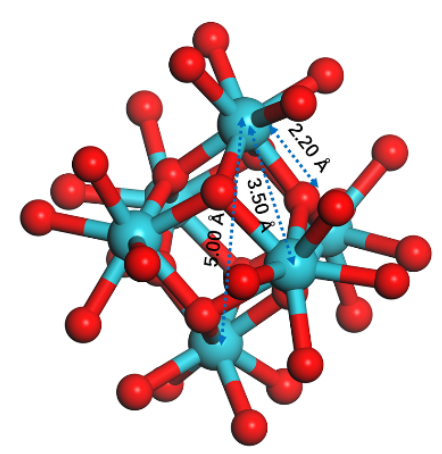

Figure 1. A) PDF for the solution of $\mathrm{ZrCl}_{4}$ in $\mathrm{DMF}$ and $\mathrm{HCl}$. Inset shows enlarged PDF for the solution of $\mathrm{ZrCl}_{4}$ in DMF-HCl fitted with hexanuclear $\mathrm{Zr}$ cluster SBU model. B) Hexanuclear Zr cluster obtained from fitting the data in A (red: oxygen atom; blue: $\mathrm{Zr}$ atom). The PDF data were modelled using DiffPyCMI. ${ }^{[20]}$

applied in order to confirm the presence of the clusters, and no modelling of solvent effects, correlated movements etc. was undertaken. Such effects contribute to a broadening of the peaks and lead to residual intensity between the model and the observed PDF. The PDF show a strong similarity to the PDF features below $7 \AA$ for amorphous and crystalline UiO-66, ${ }^{[17,21]}$ supporting that the observed cluster structure closely resemble the SBU for the UiO-66.

Previous research has systematically studied the structure of $\mathrm{Zr}(\mathrm{IV})$ multinuclear species in various solutions from different precursor salts by EXAFS, mass-spectrometry and computational studies, ${ }^{[22]}$ which indicated that zirconium shows a strong tendency toward hydrolysis and polymerization. There is a common agreement that the tetramer complex $\mathrm{Zr}_{4}(\mathrm{OH})_{8}\left(\mathrm{H}_{2} \mathrm{O}\right)_{16}$ with extraordinary stability is the dominant hydrolysis species in zirconium aqueous solution; [22a-c, 23] while the extended oligomer chains $\mathrm{Zr}_{8} \mathrm{O}_{28}\left(\mathrm{NO}_{3}\right)_{8}$ is the dominant species in $\mathrm{Zr}$ oxynitrate in methanol or $\mathrm{Zr}$ acetate aqueous solution. ${ }^{[22 \mathrm{~d}, 24]}$ In the current study, it is clear that neither the tetramer species nor the extended oligomer chains explain the higher peak area of $3.5 \AA$ than $2.2 \AA$, which shows disagreement with the experimental PDF of the dissolved $\mathrm{Zr}(\mathrm{IV})$ precursor in DMF and $\mathrm{HCl}$ solution (Figure S9S10). On the contrary, the UiO-66 SBU (hexamer) is a more suitable model and provides improved fits compared to the $\mathrm{Zr}_{4}(\mathrm{OH})_{8}\left(\mathrm{H}_{2} \mathrm{O}\right)_{16}$ and $\mathrm{Zr}_{8} \mathrm{O}_{28}\left(\mathrm{NO}_{3}\right)_{8}$ cluster (Figure S8). This indicates the predominant presence of UiO-66 SBU structures in $\mathrm{ZrCl}_{4}$ precursor solution without addition of organic ligand. This 


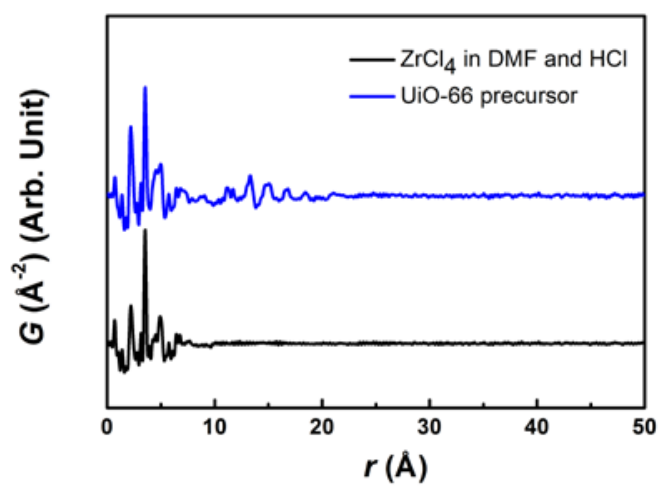

2 Figure 2. PDF comparison of the UiO-66 precursor solution (blue) and $\mathrm{ZrCl}_{4}$ in DMF-HCl (black)

4 hexanuclear zirconium cluster has previously been observed as 5 dominant structure in $\operatorname{Zr}(\mathrm{IV})$ aqueous solution with addition of 6 acetic acid $(\mathrm{pH}=1.5)$ by EXAFS. ${ }^{[22 c]}$ However, the present PDF 7 analysis is the first evidence of this SBU existing at room

8 temperature in the metal source precursor solution usually used 9 for UiO-66 synthesis.

Cluster formation in the UiO-66 precursor solution prior to heating

Next the complete UiO-66 precursor solution was studied

14 before heating by adding the organic ligand to the solution of $\mathrm{ZrCl}_{4}$

15 in DMF- $\mathrm{HCl}(\mathrm{pH}=1.8)$. This solution was stirred until transparent

16 to make the UiO-66 precursor solution, and the resulting PDF is shown in Figure 2. The observed PDF is dominated by atom-atom correlations involving the strongly scattering $\mathrm{Zr}$ atoms. Compared with the PDF of the $\mathrm{ZrCl}_{4}$ solution (Figure 2), the UiO66 precursor shows structural correlations to larger distance. In

21 the UiO-66 precursor solution the correlations extend to beyond $2220 \AA$, indicating that larger clusters form spontaneously upon
44 1.8-30 A). The higher degree of disorder at large $r$ values 45 complicates a complete refinement of the datasets (Figure $3 \mathrm{~b}$,

\section{(A)}

(B)
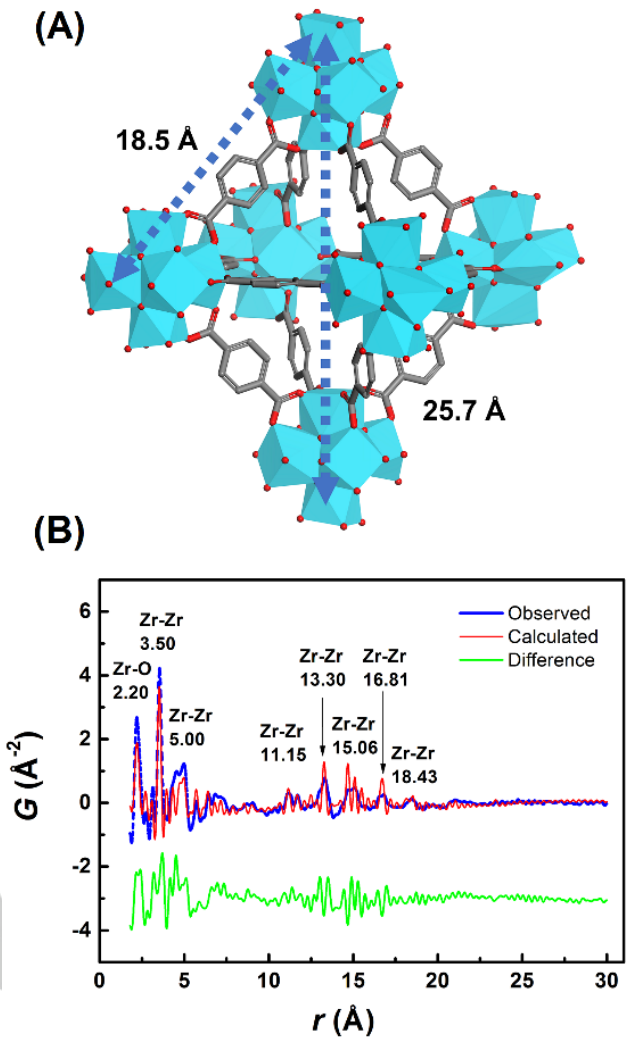

(C)

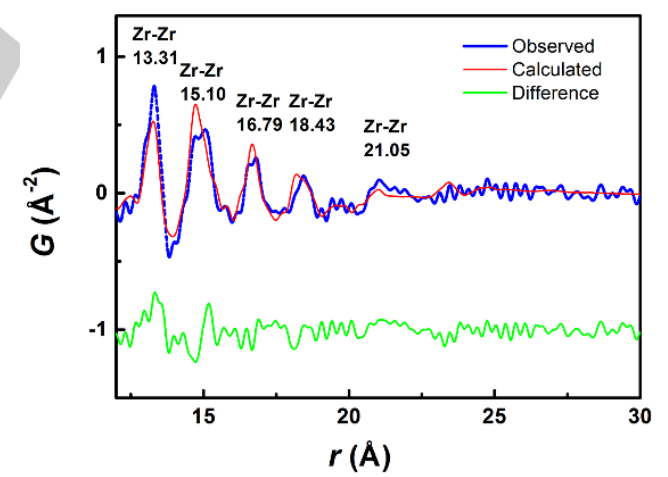

Figure 3. A) Octahedral cluster (6 SBU) present in the UiO-66 precursor solution. (red: oxygen atom; blue polyhedron: $\mathrm{Zr}$ atom) B) Full range PDF for the UiO-66 precursor solution fitted with octahedral 6 SBU cluster and fixed atomic displacement parameters (ADPs). C) High $r$ range PDF for the UiO-66 precursor solution fitted with octahedral 6 SBU cluster with refined ADPs. The high-r fit is significantly improved with refined ADPs, most likely because these account for dynamic effects such as rigid body movement of linked SBUs.

Figure S28-38 and Table S6). A high $r$ region (12-30 Å) was refined separately where the disorder was allowed to be absorbed into the atomic displacement factors (ADP). This approach gave models with a lower residual than the full range modelling (Figure 3c, Figure S39-46 and table S8). According to the high $r$ range modelling, 2-5 SBUs models provide high $R_{w}$ and unsatisfactory 
1 fits (Figure S39-42 and Table S8); while 6-9 SBUs provide9

2 reasonable fits. However they are difficult to distinguish due 60

3 experimental and modelling limitations (Figure S43-46 and Tab@1

4 S8). The similar $R_{w}$ values between the 6-9 SBUs models also

5 indicate that a cluster size distribution is present in the precursor

6 solution. Based on visual inspection of the dampening of the

7 peaks, the 6-7 SBUs are the predominant cluster sizes. Thus the

8 high $r$ range refinements suggest that the majority of the clusters

9 present are 6-7SBUs (Figure S43-44). The large ADP values

10 obtained in the refinements confirm that a substantial degree of

11 disorder is present in the clusters.

12 Nevertheless residual intensity is still present between the

13 model and the experimental PDF. This can largely be explained

14 by two factors. Firstly, the distribution of cluster sizes and shape

15 will provide changes in the relative ratio between the calculated

16 PDF peaks, which may cause residual intensity. Secondly, the

17 peaks in the observed PDF are considerably broader than the

18 model peaks (Figure S49), which also lead to residual intensity.

19 The broader peaks of the observed PDF compared to the model

20 also suggests substantial degree of disorder in the cluster

21 compared with the final UiO-66 crystal structure obtained from

22 data on a reference powder sample. ${ }^{[19]}$ It is interesting to compare

23 the observed PDF with the pioneering work by Bennett et al. and

24 Chapman et al. on PDF for both amorphous and crystalline UiO-

$2566 .{ }^{[17,21]}$ The PDF features below $7 \AA$ is very similar with both

26 amorphous and crystalline UiO-66, ${ }^{[17,21]}$ supporting that the

$27 \mathrm{Zr}_{6}(\mathrm{O})_{4}(\mathrm{OH})_{4} \mathrm{SBU}$ structure keep intact, and a limited structural

28 distortion is observed; while the PDF features above $7 \AA$ show

29 differences with that of both amorphous and crystalline UiO-66.

30 These peaks represent the structural features obtained by the

31 linkage of SBUs. For the PDF features above $7 \AA$, the observed

32 PDF exhibits longer range order than the amorphous UiO-66 [21]

33 and the peak signal damping is significantly slower supporting that

34 in the observed PDF the SBUs are linked by the ligands, in

35 contrast to the partial isolated SBUs in the amorphous UiO-66.

36 The observed PDF have broader peaks with lower intensity than

37 the crystalline UiO-66, ${ }^{[21]}$ equally, the peak signal damping is

38 much faster supporting that there is a substantial degree of

39 disorder in the cluster at this stage compared with the well-

40 crystalized UiO-66. In agreement with previous studies on defect

41 MOFs, ${ }^{[10 \mathrm{~b}, 25]}$ we speculate that the clusters, while resembling the

42 UiO-66, still exhibit significant disorder features such as missing

43 ligands and/or are in wrong coordination mode compared with th 62

44 perfect UiO-66 crystal structure. ${ }^{[25 b]}$ Additionally, rigid-body

45 motion of the cluster SBUs in solution will also broaden the peales

46 in the PDF.

47 Previously several studies have used SAXS, EXAFS, ESI-MS

48 or NMR to demonstrate the formation of multinuclear clusters prigr6

49 to MOF crystallization. ${ }^{[10 b, ~ c, ~ 13 a, ~ 18, ~ 26] ~ F o r ~ e x a m p l e, ~ i n ~ s i t u ~ S A X ~} \$ 7$

50 studies of ZIF-8 have identified the spontaneous formation ff

51 clusters with approximately sizes of $20 \AA$ when mixing theg

52 precursor component solution. ${ }^{[10 c]}$ However, SAXS cannot 0

53 provide atomic level structural information, and the study on $/ 1$

54 proposed the initial clusters as monodisperse homogeneout $\$ 2$

55 spheres while the internal atomic structure of the cluster remained 3

56 unknown. Recently, a pioneering in situ PDF study also revealed 4

57 a high concentration of $\mathrm{Zn}(2-\text { Melm })_{4}$ cluster initially formed and 5

58 remained stable over long times before crystallization during $Z \mathrm{ZF}_{6}$ formation. ${ }^{[18]}$ Thus, this present study provides a direct structural evidence of the atomic structure of MOF prenucleation clusters.

A)

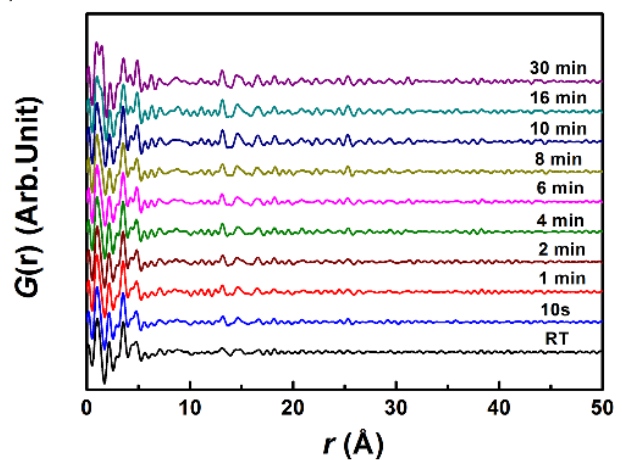

B)

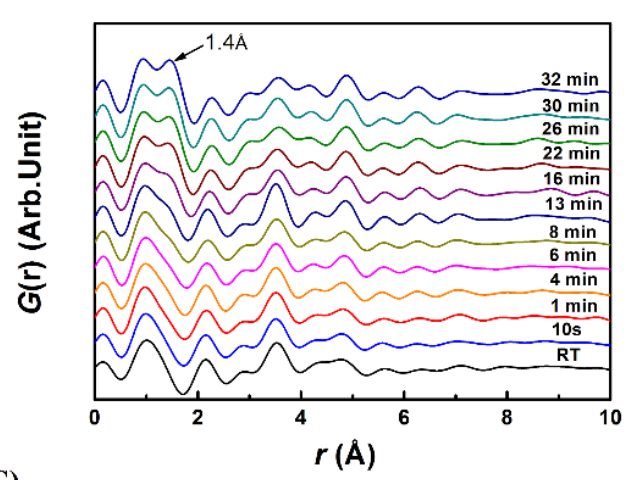

C)

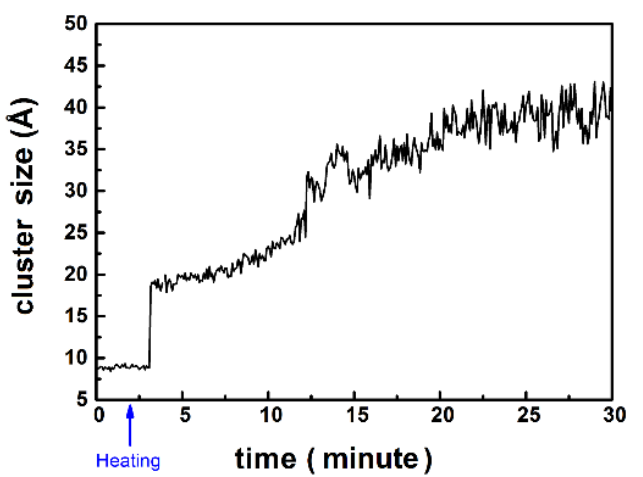

Figure 4. A) Selected PDFs illustrating the reaction progress at $150^{\circ} \mathrm{C}$. B) Magnified PDFs shows the evolution of PDF at $1.4 \AA$ with heating time at $150^{\circ} \mathrm{C}$. C) The evolution of the cluster size with time.

\section{Crystal growth and ordering upon heating}

Figure 4a shows the evolution of the PDF with respect to time when heating at $150^{\circ} \mathrm{C}$. The structure in solution maintains the same short-range coordination distances before and after the heating is initiated. It is worthy to note that in the short range PDF, the main atom distances of $\mathrm{Zr}-\mathrm{O}, \mathrm{Zr}$ - $\mathrm{Zr}$ and $\mathrm{Zr}$ - $\mathrm{Zr}$ remained at 2.2, 3.5 and $5.0 \AA$, respectively, and no change of main atom distances were found, indicating that there is limited distortion of SBU structure during crystallization. ${ }^{[17]}$ In the long range PDF, peaks arise and grow in the regions around $r=15.0 \AA$ and $r=$ $25.0 \AA$, corresponding to inter-SBU distances in the UiO-66 
1 crystal structure, which indicates the assembly of clusters in th 60

2 precursor solution. The refined maximum correlation lengt 1

3 increases quickly after heating is applied revealing that the clust 62

4 growth is rapid (Figure 4c). With increasing heating time, the

5 growth rate of the cluster slows down, and the PDF peaks become

6 more well-defined.

7 After 4 minutes, a very small peak (firstly as a shoulder) at 1.4

8 A begins to appear and gradually becomes more well defined and

9 increase in intensity, as shown in magnification in Figure $4 \mathrm{~b}$ (see

10 also Figure S51). This distance is specific for the $\mathrm{C}-\mathrm{N}$ and

11 aromatic $\mathrm{C}-\mathrm{C}$ bond in the ligand. This possibly can be ascribed to

12 ligands migrating from the solvent and into the UiO-66 structure,

13 and due to the higher density in the crystal compared to the

14 solvent, the $1.4 \AA$ peak increases in intensity. The in situ reaction

15 cell is not uniformly heated, meaning that the non-heated volume

16 will act as a precursor reservoir from which the ligands might

17 diffuse. At the same time the as-formed particles transform from

18 their initial disordered state, which was also indicated by the initial

19 broadening of the peaks, to a cluster that represent the UiO-66

20 structure more correctly with more well-defined PDF peaks. After

214 minutes, the entire PDF pattern gradually resembles the pattern

22 from the UiO-66 crystal structure (Figure 4a). The high-r features

23 continue to increase in intensity throughout the experiment, and

24 this shows how full crystallization and ordering of the structure

25 requires extended time at elevated temperature.

26 Due to the limitation of instrumental conditions, the noise in the

27 in situ RT data at long correlation distances ( $>10 \AA)$ is too high to

get a precise estimate of the particle size. However, even if the

29 absolute value of the particle size obtained from the PDF fitting

30 should be interpreted with caution, then the trend in the crystal

31 growth should be correct. Figure $4 \mathrm{c}$ reveals the evolution of the

32 relative growth of the cluster size over time, and a gradug/4

33 increase in cluster size is observed following a sudden jump 65

34 approximately $70 \mathrm{~s}$ after initiation of heating has commence $\$ 6$

35 This can be ascribed to the fast aggregation of the multinuclear

36 clusters in UiO-66 precursor solutions. It is worth to mention th 97

37 the aggregation process has also been observed to be exist 68

38 an independent process before fully crystallization, based on th 69

39 SAXS, WAXS and DFT analysis by the groups of Goesten and0

40 Gascon. ${ }^{[10 b]}$ A slight increase in the cluster size is observed1

41 beyond the last measurement at about $32 \mathrm{~min}$. Modeling of the 2

42 PDF obtained at the last measurement with the final UiO- 663

43 crystal structure gives a satisfactory fit (Figure S527,4

44 demonstrating successful synthesis of UiO-66 nanocrystals 5

during the in situ synchrotron experiment.

76 To further analyze the UiO-66 crystallization process we7 performed synthesis using the same precursor in a reactor that 8 mimics the in situ conditions (Figure 5). ${ }^{[27]}$ Three main features9 were obtained from Rietveld refinement of these PXRD pattern\$. Firstly, Rietveld refinements of the PXRD of the sample1 synthesized at $150{ }^{\circ} \mathrm{C}$ for 60 minutes agrees well with the2 crystallographic database ${ }^{[19]}$ (Figure $5 b$ ), which also verifies the

53 successful synthesis of UiO-66 crystalline nanoparticle8.4

54 Secondly, the 3 min sample contains both the characteristic Bragg 5

55 peaks for UiO-66 and some very broad peaks, which indicates the 6

56 coexistence of crystalline particles and clusters that are yet 87

57 assemble into crystalline particles. And thirdly, the PXR 88

58 crystalline size of the $3 \mathrm{~min}$ and $60 \mathrm{~min}$ samples are $3.4 \mathrm{~nm}$ ang 9 with the PDF refinement results where the cluster size first quickly increases followed by a slower growth during which the particles undergo structural ordering.

(A)

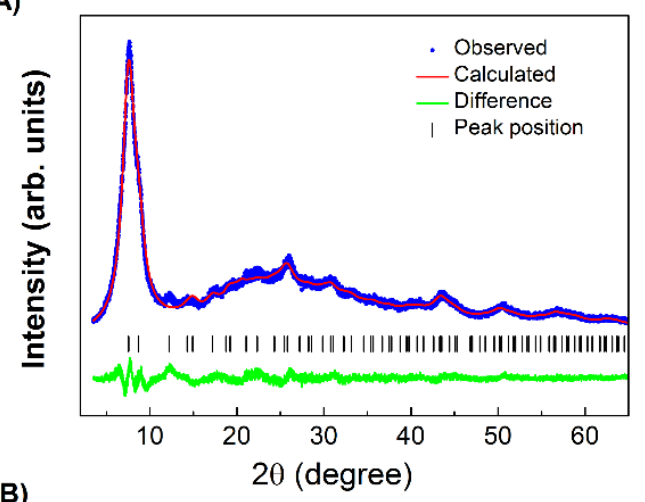

(B)

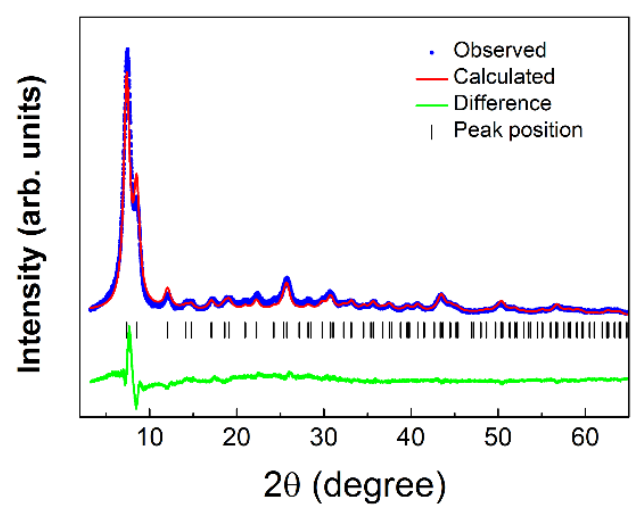

Figure 5. Rietveld refinement of PXRD data of UiO-66 synthesized using the same precursor solution using a synthesis reactor that mimics the in situ conditions, ${ }^{[27]}$ at $150^{\circ} \mathrm{C}$ for (A) 3 min. (B) $60 \mathrm{~min}$.

\section{Summary: Formation mechanism of UiO-66}

Overall, the UiO-66 formation process can be described in four steps:

- $\quad$ Step 1, The hexanuclear SBU structure is formed in the $\mathrm{ZrCl}_{4}$ metal salt precursor solution

- $\quad$ Step 2, Assembly of multinuclear clusters containing SBUs takes place with addition of organic ligand in UiO66 homogenous precursor solution

- $\quad$ Step 3, The multi-SBU clusters quickly aggregate soon after heating is applied

- $\quad$ Step 4, The UiO-66 structure undergoing ordering and growth gradually become fully crystalline.

Figure 6 summarizes the species detected by the PDF and PXRD analysis from the initial precursor cluster to the formation of final UiO-66 nanocrystal.

The aggregation of large molecular clusters with subsequent internal ordering is highly complex, and not described by classical nucleation theory, where thermodynamic energy is understood to lead to a critical nucleus size in a supersaturated solution. The observed nucleation of UiO-66 exhibits similarities with the prenucleation clusters (PNC) model, where "the PNCs are amorphous or nano-crystalline assemblies of atoms in a structurally stable, yet soluble entity". ${ }^{[28]}$ The precursor cluster in 
1 UiO-66 is very large at approximately $2.3 \mathrm{~nm}$, which is almost one

2 unit cell of the final crystal structure.
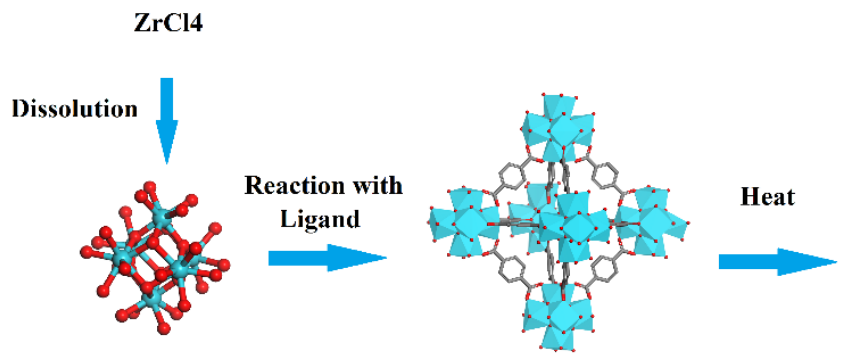

Step 1:

Step 2:

Formation of SBU $\quad$ Formation of multinuclear cluster

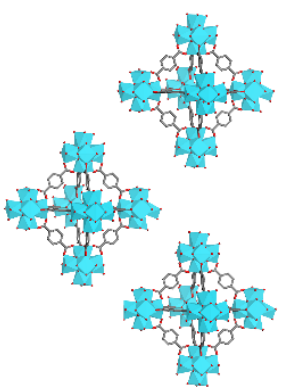

Step 3: Cluster aggregation

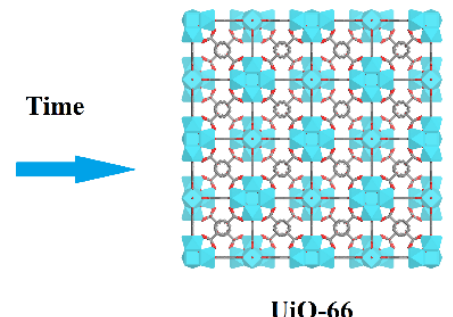

Step 4: Structure ordering

Figure 6. The structure of the species detected by PDF during nucleation and early growth of UiO-66 nanoparticle

\section{Conclusion}

\section{Experimental Section}

$18 \mathrm{Zr}(\mathrm{IV})$ in DMF-HCl solution: $\mathrm{ZrCl}_{4}(0.45 \mathrm{~g}, 1.93 \mathrm{mmol})$ (Sigmaz 19 Aldrich, $\geq 99.9 \%$ ) was dissolved in N,N'- dimethylformamide (DMF' (8.3 mL, $13.7 \mathrm{mmol}$ ) (Sigma Aldrich, $\geq 99 \%)$ and $\mathrm{HCl}(1.66 \mathrm{mg} 7$

21 (Sigma Aldrich, 37 wt. \% in $\mathrm{H}_{2} \mathrm{O}$ ) at room temperature, anel8

22 magnetically stirred for an hour to obtain a transparent solutiop9 $(\mathrm{pH}=1.8)$. Descriptions of the preparations of the other $\mathrm{Zr}(\mathrm{I} G \mathrm{O}$ solutions are provided in supporting information.

UiO-66 precursor solution: The synthesis procedure is base 62

26 upon the study of Cavka and Lillerud, ${ }^{[19]}$ with changes suggested

27 by Serre et al..$^{\left[{ }^{[c]}\right]}$ and Gascon et al.$^{[10 \mathrm{bb}]} \mathrm{A}$ high concentration of the

28 precursor is used in order to obtain high PDF signal, and a hig $\$ 3$

29 heating temperature was used in order to speed up the reaction.

$3010 \mathrm{~mL} \mathrm{Zr(IV)}$ in DMF-HCl solution was prepared the same way d 4

31 the above. Then 2-Aminoterephthalic Acid (2- $\mathrm{NH}_{2}-\mathrm{BDC}, 0.33 €, 5$
$321.82 \mathrm{mmol}$ ) (Sigma Aldrich, 99\%) was added into the solution, and 33 magnetically stirred for about an hour to obtain a transparent 34 yellow solution $(\mathrm{pH}=1.8)$

Total X-ray scattering measurements: The ex situ total scattering measurements of different $\mathrm{Zr}(\mathrm{IV})$ solutions were carried out at beamline ID-31 at ESRF, Grenoble. The solutions were loaded in kapton capillaries (diameter $=1 \mathrm{~mm}$ ). The in situ total scattering PDF experiments were performed at beam-line P02.1 at PETRAIII, DESY, Germany. The in situ total scattering measurement of UiO-66 synthesis was carried out by loading the UiO-66 precursor solution into a fused silica capillary reactor pressurized to 103 bar and heated to temperature $150{ }^{\circ} \mathrm{C}$. The experimental setup used for in situ total scattering has been described by Becker et al. ${ }^{[29]}$

Data treatment: The raw two-dimensional data was integrated with the program Dioptas ${ }^{[30]}$ to obtain total scattering data. Then the total scattering structure factor $S(Q)$ were Fourier transformed using the $x P D F s u i t e$ software. ${ }^{[31]}$ Prior to the Fourier transformation, the data were corrected for background scattering using measurements corresponding to pure solvents at identical conditions. The resulting PDF for different $\mathrm{Zr}(\mathrm{IV})$ solutions and UiO-66 precursor solution were refined using the Diffpy-CMI software based on the Debye function. ${ }^{[20]}$ The UiO-66 synthesis in situ PDFs were refined sequentially using the PDFgui software, which is based on the crystallographic approach. The structural refinement of the aqueous $\mathrm{Zr}(\mathrm{IV})$ solution is based on crystallographic data of tetragonal $\left[\mathrm{Zr}_{4}(\mathrm{OH})_{8}\left(\mathrm{OH}_{2}\right)_{16}\right]^{8+}$ from ICSD27437. ${ }^{[22 c]}$ The structural refinement of UiO-66 precursor and UiO66 is based on crystallographic data from ICSD-4512072. ${ }^{[19]}$ The PXRD data were refined using Rietveld method in the FullProf Suite. ${ }^{[32]}$

\section{Acknowledgements}

Steinar Birgisson, Lirong Song, Dipankar Saha, Aref Hasen Mamakhel, Ann-Christine Dippel, Hazel Readon, Yin Hao and 


\section{Ful1 Paper}

\section{Notes and references}

10 Throughout the article, the term "crystallite" will be used describe atoms which assemble into long range structures, whi

Mirijam Zobel are thanked for fruitful discussions and assistanç1 with the synthesis, measurements and analysis. This work ts supported by the Danish National Research Foundation 4 (DNRF93). H. X. acknowledge financial support from the Nations Natural Science Foundation of China (51602301). The ESRF and PETRAIII synchrotron facilities are sincerely thanked for beam8 time. Affiliation with the Aarhus University Center for Integrated Materials Research (iMAT) is gratefully acknowledged. "particles" is used to describe both the crystalline as well as th amorphous structure of an assembly of atoms. "Clusters" is used to describe small, non-random units of atoms, such as the ores displayed in Figure 1. The terms "precursor cluster" "prenucleation cluster" are used to describe the clusters form 46 in a precursor solution at room temperature. In this article the presumed MOF multi-SBUs should be understood as 99 prenucleation cluster

a)S. Kitagawa, Chem. Soc. Rev. 2014, 43, 5415-5418; b)H. Kim Yang, S. R. Rao, S. Narayanan, E. A. Kapustin, H. Furukawa, At Umans, O. M. Yaghi, E. N. Wang, Science 2017, 356, 430-434; Furukawa, K. E. Cordova, M. O'Keeffe, O. M. Yaghi, Science 20 341, 1230444; d)A. Cadiau, K. Adil, P. Bhatt, Y. Belmabkhout, Eddaoudi, Science 2016, 353, 137-140. a)S. Yuan, L. Feng, K. Wang, J. Pang, M. Bosch, C. Lollar, Y.
J. Qin, X. Yang, P. Zhang, Adv. Mater. 2018; b)C. S. Diercks, Y K. E. Cordova, O. M. Yaghi, Nat. Mater. 2018, 1; c)M. O'Keeffe A. Peskov, S. J. Ramsden, O. M. Yaghi, Acc. Chem. Res. 2008, 1782-1789. a)N. D. Loh, S. Sen, M. Bosman, S. F. Tan, J. Zhong, C. A. Nijhuli P. Král, P. Matsudaira, U. Mirsaidov, Nat. Chem. 2016; b)J. J. Yoreo, N. A. Sommerdijk, Nature Reviews Materials 2016, 1, 16 H. Mistry, A. S. Varela, S. Kühl, P. Strasser, B. R. Cuenya, Naü

[5] Reviews Materials 2016, 1, 16009 . from air pollution to climate change, John Wiley \& Sons, 2016. a)G. M. Pound, V. K. L. Mer, J. Am. Chem. Soc. 1952, 74, 23 2332; b)M. Avrami, The Journal of Chemical Physics 1939, 7, 1 1112; c)J. De Yoreo, Nat. Mater. 2013, 12, 284-285.

R. I. Walton, F. Millange, The Chemistry of Metal? Organive Frameworks: Synthesis, Characterization, and Applicationg. Synthesis, Characterization, and Applications 2016, 729-764. a)J. Zhao, B. Kalanyan, H. F. Barton, B. A. Sperling, G. N. Pars Chem. Mater. 2017, 29, 8804-8810; b)D. Zacher, R. Schmid, C. W\% R. A. Fischer, Angew. Chem. Int. Ed. 2011, 50, 176-199; c)R. W I. Strashnov, M. W. Anderson, M. P. Attfield, Angew. Chem. Int. 2016, 55, 9075-9079; d) J. P. Patterson, P. Abellan, M. S. Dennȳ З C. Park, N. D. Browning, S. M. Cohen, J. E. Evans, N. C. Giannedchi6 J. Am. Chem. Soc. 2015, 137, 7322-7328; e)J. Cravillon, R. Nayjuß,7 S. Springer, A. Feldhoff, K. Huber, M. Wiebcke, Chem. Mater. 20138 23, 2130-2141; f)D. Biswal, P. G. Kusalik, ACS nano 2016, 11, 2 ;g

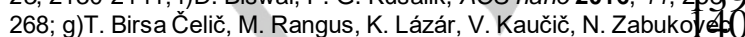
Logar, Angew. Chem. Int. Ed. 2012, 51, 12490-12494; h)R. Amelodt F. Vermoortele, J. Hofkens, F. C. De Schryver, D. E. De Vos, M 42 F. Vermoortele, J. Hofkens, F. C. De Schryver, D. E. De Vos, M 142
Roeffaers, Angew. Chem. Int. Ed. 2013, 52, 401-405. a)H. H. M. Yeung, Y. Wu, S. Henke, A. K. Cheetham, D. O'Hare 44 I. Walton, Angew. Chem. 2016, 128, 2052-2056; b)Y. Wu, M.45 Breeze, G. J. Clarkson, F. Millange, D. O'Hare, R. I. Walton, Ang 4 Chem. Int. Ed. 2016, 55, 4992-4996; c)F. Ragon, P. Horcajada 47 Chevreau, Y. K. Hwang, U.-H. Lee, S. R. Miller, T. Devic, J]Chang, C. Serre, Inorg. Chem. 2014, 53, 2491-2500; d)F. Millanild M. I. Medina, N. Guillou, G. Férey, K. M. Golden, R. I. Walitos, Angew. Chem. Int. Ed. 2010, 49, 763-766; e)P. A. Julien 1 K.1 Užarević, A. D. Katsenis, S. A. Kimber, T. Wang, O. K. Farha, 1 Zhang, J. Casaban, L. S. Germann, M. Etter, J. Am. Chem. \$宁2
2016, 138, 2929-2932.
a)E. Stavitski, M. Goesten, J. Juan - Alcañiz, A. Martinez - Joaristi P. Serra - Crespo, A. V. Petukhov, J. Gascon, F. Kapteijn, Angew. Chem. Int. Ed. 2011, 50, 9624-9628; b)M. Goesten, M. De Lange, A. Olivos-Suarez, A. Bavykina, P. Serra-Crespo, C. Krywka, F. Bickelhaupt, F. Kapteijn, J. Gascon, Nat. Commun. 2016, 7; c)J. Cravillon, C. A. Schröder, R. Nayuk, J. Gummel, K. Huber, M. Wiebcke, Angew. Chem. 2011, 123, 8217-8221.

[11] A. Schoedel, O. M. Yaghi, The Chemistry of Metal? Organic Frameworks: Synthesis, Characterization, and Applications: Synthesis, Characterization, and Applications, 41-72.

[12] a)A. Schoedel, M. J. Zaworotko, Chem. Sci. 2014, 5, 1269-1282 b)M. Bosch, S. Yuan, W. Rutledge, H.-C. Zhou, Acc. Chem. Res. 2017, 50, 857-865.

[13] a)S. Surble, F. Millange, C. Serre, G. Ferey, R. I. Walton, Chem Commun. 2006, 1518-1520; b)M. Haouas, C. Volkringer, T. Loiseau, G. r. Férey, F. Taulelle, Chem. Mater. 2012, 24, 2462-2471; c)G. r. Férey, M. Haouas, T. Loiseau, F. Taulelle, Chem. Mater. 2014, 26, 299-309.

[14] M. Shoaee, M. W. Anderson, M. P. Attfield, Angew. Chem. 2008 120, 8653-8656.

[15] T. Egami, S. J. Billinge, Underneath the Bragg peaks: structural analysis of complex materials, Vol. 16, Elsevier, 2003

[16] a)M. Zobel, R. B. Neder, S. A. Kimber, Science 2015, 347, 292-294; b)D. Saha, K. M. Jensen, C. Tyrsted, E. D. Bøjesen, A. H. Mamakhel, A. C. Dippel, M. Christensen, B. B. Iversen, Angew. Chem. Int. Ed. 2014, 53, 3667-3670; c)P. Nørby, S. Johnsen, B. B. Iversen, ACS nano 2014, 8, 4295-4303; d)K. M. Jensen, M. Christensen, P. Juhas, C. Tyrsted, E. D. Bøjesen, N. Lock, S. J. Billinge, B. B. Iversen, J. Am. Chem. Soc. 2012, 134, 6785-6792; e)E. D. Bøjesen, K. M. Jensen, C. Tyrsted, A. Mamakhel, H. L. Andersen, H. Reardon, J. Chevalier, A.-C. Dippel, B. B. Iversen, Chem. Sci. 2016, 7, 6394 6406.

[17] A. E. Platero-Prats, A. Mavrandonakis, L. C. Gallington, Y. Liu, J. T. Hupp, O. K. Farha, C. J. Cramer, K. W. Chapman, J. Am. Chem. Soc 2016, 138, 4178-4185.

[18] M. W. Terban, D. Banerjee, S. Ghose, B. Medasani, A. Shukla, B. A. Legg, Y. Zhou, Z. Zhu, M. L. Sushko, J. J. De Yoreo, Nanoscale 2018, 10, 4291-4300

[19] J. H. Cavka, S. Jakobsen, U. Olsbye, N. Guillou, C. Lamberti, S. Bordiga, K. P. Lillerud, J. Am. Chem. Soc. 2008, 130, 13850-13851. [20] P. Juhás, S. Billinge, Foundations of Crystallography 2017, 73, a388 [21] T. D. Bennett, T. K. Todorova, E. F. Baxter, D. G. Reid, C. Gervais B. Bueken, B. Van de Voorde, D. De Vos, D. A. Keen, C. MellotDraznieks, Physical Chemistry Chemical Physics 2016, 18, 21922201

[22] a)C. Walther, J. Rothe, M. Fuss, S. Büchner, S. Koltsov, T. Bergmann, Anal Bioanal Chem 2007, 388, 409-431; b)N. Rao, M. N. Holerca, V. Pophristic, J Chem Theory Comput 2008, 4, 145-155; c)C. Hennig, S. Weiss, W. Kraus, J. Kretzschmar, A. C. Scheinost Inorg. Chem. 2017, 56, 2473-2480; d)A.-C. Dippel, K. Jensen, C Tyrsted, M. Bremholm, E. D. Bøjesen, D. Saha, S. Birgisson, M. Christensen, S. J. Billinge, B. B. Iversen, Acta Crystallographica Section A: Foundations and Advances 2016, 72, 645-650.

[23] A. Clearfield, P. A. Vaughan, Acta Crystallographica 1956, 9, 555 558.

[24] C. Tyrsted, N. Lock, K. Jensen, M. Christensen, E. D. Bøjesen, H Emerich, G. Vaughan, S. J. Billinge, B. B. Iversen, IUCrJ 2014, 1 165-171.

[25] a)T. D. Bennett, A. K. Cheetham, Acc. Chem. Res. 2014, 47, 1555 1562; b)G. C. Shearer, S. Chavan, S. Bordiga, S. Svelle, U. Olsbye K. P. Lillerud, Chem. Mater. 2016, 28, 3749-3761.

[26] a)G. r. Férey, M. Haouas, T. Loiseau, F. Taulelle, Chem. Mater. 2013, 26, 299-309; b)I. H. Lim, W. Schrader, F. Schüth, Chem Mater. 2015, 27, 3088-3095

[27] C. Granados-Miralles, M. Saura-Múzquiz, E. D. Bøjesen, K. M. Jensen, H. L. Andersen, M. Christensen, J. Mater Chem. C 2016 4, 10903-10913.

[28] a)D. Gebauer, M. Kellermeier, J. D. Gale, L. Bergström, H. Cölfen Chem. Soc. Rev. 2014, 43, 2348-2371; b)E. D. Bøjesen, B. B. Iversen, Crystengcomm 2016, 18, 8332-8353.

[29] J. Becker, M. Bremholm, C. Tyrsted, B. Pauw, K. Jensen, J. Eltzholt, M. Christensen, B. B. Iversen, J. Appl. Crystallogr. 2010, 43, 729736.

[30] C. Prescher, V. B. Prakapenka, High Pressure Research 2015, 35 , 223-230.

[31] P. Juhás, T. Davis, C. L. Farrow, S. J. Billinge, J. Appl. Crystallogr 2013, 46, 560-566.

[32] J. Rodríguez-Carvajal, Physica B: Condensed Matter 1993, 192, 55 69. 


\section{Entry for the Table of Contents}

\section{COMMUNICATION}

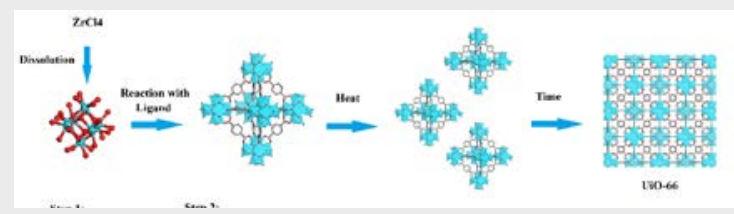

Hui Xu, Sanna Sommer, Nils Lau Nyborg Broge, Junkuo Gao and Bo. B. Iversen*

Page No. - Page No.

The Chemistry of Nucleation: in situ Pair Distribution Function analysis of secondary building units during UiO-66 MOF formation

In situ PDF analysis was used to reveal the atomic scale nature of precursor species during solvothermal UiO-66 MOF synthesis, and a hexanuclear zirconium cluster was established as the secondary building unit present even in the metal salt precursor solution. 